\title{
IMPROVEMENTS IN SEPARATION OF NON-FERROUS SCRAP METALS USING AN ELECTROMAGNETIC SENSOR
}

\author{
M.B. MESINA ${ }^{\mathrm{a}}$, T.P.R. DE JONG ${ }^{\mathrm{a}, *}$ and W.L. DALMIJN ${ }^{\mathrm{b}}$ \\ ${ }^{a}$ Department of Applied Earth Sciences, Faculty of Civil Engineering and Geosciences, \\ Mijnbouwstraat 120, 2628 RX, Delft, The Netherlands; ${ }^{b}$ Faculty of Design, Construction and \\ Production, Mekelweg 2, 2628 CD, Delft, The Netherlands
}

(Received 30 December 2002; In final form 27 January 2003)

\begin{abstract}
This article describes a new method for identification and separation of non-ferrous scrap metals using an electromagnetic sensor that is based on the eddy current principle. The electromagnetic sensor (EMS) is a prototype system that has been developed by Delft University of Technology in co-operation with industry. Experimental results obtained with different non-ferrous metals are presented. The theory behind the sensor and the main components of the system are described.
\end{abstract}

Keywords: Electromagnetic sensor; Eddy current; Non-ferrous metals; Separation; Sorting

\section{INTRODUCTION}

In recent years, applied researchers have become increasingly interested in developing new advanced techniques for the sorting of different types of scrap metals. One of the multiple steps in the recycling of non-ferrous metals is the separation of shredded material into different groups. After shredding, which liberates different metals from the end-of-life products, e.g. cars, home appliances, industrial waste, particles will have a small size ranging from 15 to $120 \mathrm{~mm}$.

At present, mechanical separation and manual sorting are widely used in recycling technology. Manual sorting as well as mechanical systems such as eddy current separators (ECS), heavy media separation systems (HMS) or magnets are used to separate metal particles into several fractions. However, manual sorting is labour-intensive and mechanical systems often produce insufficient product quality. Therefore, new methods are being developed to improve the separation process.

Sensors potentially improve the separation process. The improved measuring of particle properties such as colour, texture, morphology, conductivity and others, allows high quality sorting of mixed materials into pure products with significant added value. Recently, several systems based on sensor technology have entered the market for metal sorting [1].

*Corresponding author. E-mail: T.P.R.dejong@ta.tudelft.nl 


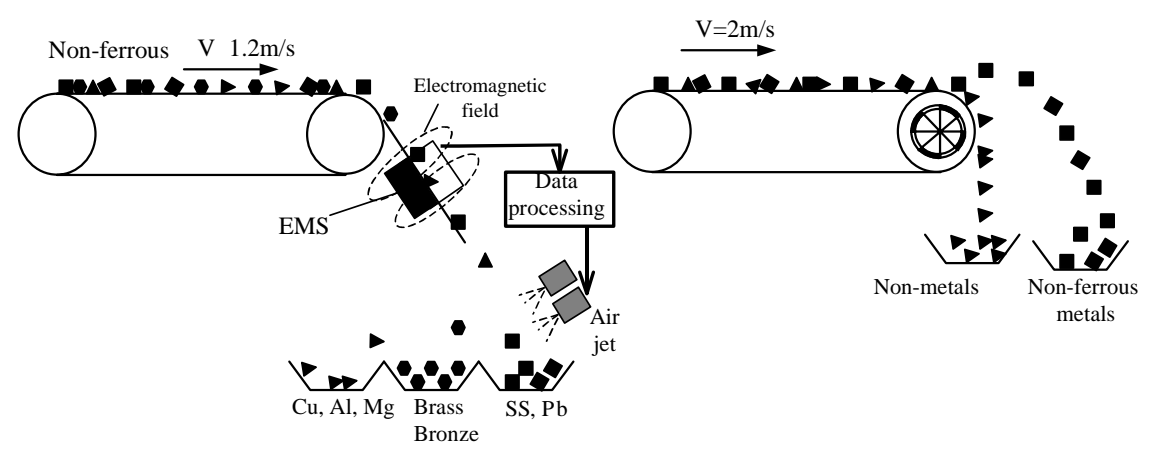

(a)

(b)

FIGURE 1 (a) EMS based on the eddy current principle; (b) the ECS uses mechanical forces for metal separation.

An automated system able to separate aluminium alloys by laser-induced brake-down spectroscopy (LIBS) is described by Rosenfeld [2]. Colour sorting is used in nonferrous metal processing with good results for separation of copper and brass [3].

New systems using X-ray dual energy transmission have been investigated at Delft University of Technology and tested for scrap processing. Satisfactory results have been obtained for separation of heavy metals from light metals [4]. However, low-cost systems with satisfactory separation results are still needed for other applications.

Despite of the wealth of information the sensors provide, there are limitations in obtaining the desired results due to the fact that only a few properties are measured. In most cases one sensor measures only one property of the inspected particle and seldom two or more. Research has been conducted at Delft University of Technology for developing sensors able to measure the electrical properties of metals. Electromagnetic sensors (EMS) are an example and such sensors can be used to improve the separation process between non-ferrous metals [5].

An EMS uses the interaction between the metals and an alternating magnetic field as a criterion for the distinction among non-ferrous metals, while for metal separation an ECS uses mechanical forces that are induced in particles. Sensing elements are used for the EMS to determine the electrical properties of metal particles. Figure 1 shows a schematic view of the EMS based on the eddy current principle and the ECS.

This article describes the design, set-up and the results of an EMS prototype developed at Delft University of Technology. The EMS is able to identify scrap non-ferrous metals and to separate them into different groups based on differentiation in electrical conductivity. The newly developed sensors have the potential to improve the quality of recycled materials at a throughput of several tonnes per hour.

\section{BACKGROUND THEORY OF THE ELECTROMAGNETIC SENSOR}

An EMS comprises two main elements, i.e. (1) a transmitter coil and (2) a receiver coil. The transmitter coil creates an alternating electromagnetic field that interacts with the metal particle placed close to the coil. This interaction is measured using the receiver coil from which a signal is obtained. In the applied experimental set-up the transmitter 


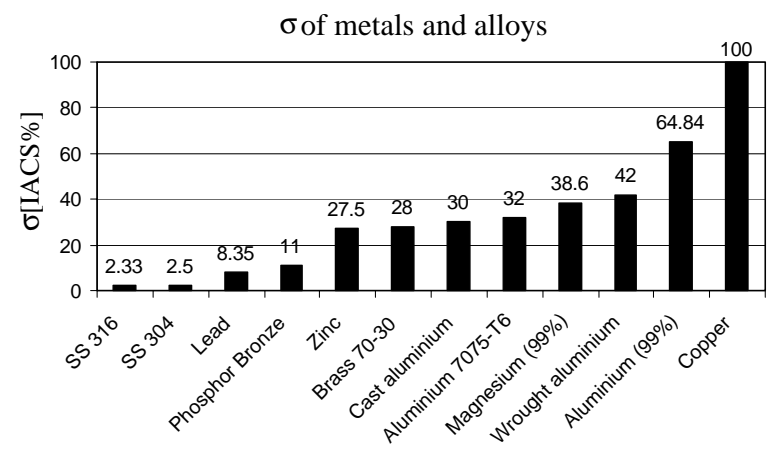

FIGURE 2 The electrical conductivity $(\sigma)$ of some common metals and alloys (IACS - International Annealed Copper Standard).

coil is excited by an alternating voltage supply ( $40 V_{\mathrm{p}-\mathrm{p}}$ - peak-to-peak) at a variable frequency between $700 \mathrm{~Hz}$ and $5 \mathrm{kHz}$.

The electrical conductivity $(\sigma)$ is a specific material property that allows the conduction of an electric current. In Fig. 2 an example of the variation in $\sigma$ for several metals is given. $\sigma$ is defined at a temperature of $20^{\circ} \mathrm{C}$. As can be seen, metals can be classified based on their electrical properties using $\sigma$ as a criterion.

\section{Generation and Detection of the Eddy Currents}

The magnetic field strength $H$ is responsible for a magnetic flux density $B$, and a magnetic flux $\phi$, which is defined as

$$
\phi=\int B \cdot n d a
$$

i.e., the integral of the normal component of $B$ over the relevant area $a$. The oscillating nature of the flux induces circulatory (eddy) currents in the material placed beneath the coil. Their direction is such that their own magnetic field opposes the field that produces them (Lenz's law). They spread out into the part, but will naturally be constrained by its boundaries. Being circulating currents, they produce their own secondary magnetic flux $\phi_{\mathrm{s}}$ opposed to $\phi_{\mathrm{p}}$. The receiver coil senses an equilibrium flux $\phi_{\mathrm{E}}$, which is the difference between $\phi_{\mathrm{p}}$ and $\phi_{\mathrm{s}}$ (Fig. 3(a)).

The receiver coil provides a voltage $U$ proportional to the flux created by the transmitter coil. The flux can be linked to $H$ and therefore $U$ can be written as:

$$
U=N_{\mathrm{s}}\left(\pi D^{2} / 4\right)(2 \pi f) \mu_{0} \mu_{\mathrm{r}} H
$$

where $N_{\mathrm{s}}$ is the number of windings of the receiver coil, $D$ its diameter, $f$ the frequency of the magnetic field, $\mu_{0}$ the vacuum magnetic permeability $\left(\mu_{0}=4 \pi \times 10^{-7} \mathrm{H} / \mathrm{m}\right)$ and $\mu_{\mathrm{r}}$ the relative magnetic permeability, which is specific for each metal. For most non-ferrous metals we have $\mu_{\mathrm{r}} \approx 1$ [6]. 


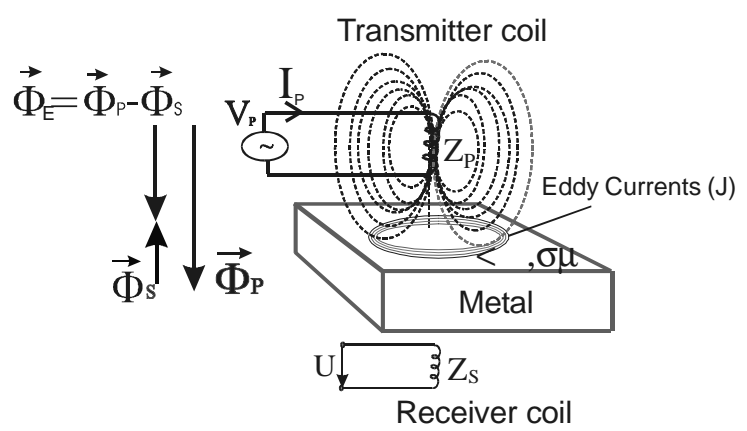

(a)

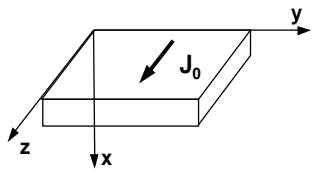

(b)

FIGURE 3 (a) Measurement principle of EMS; (b) co-ordinates for the calculation of the effective depth of penetration $\delta_{\mathrm{e}}$.

\section{Nature of the Eddy Currents}

The eddy currents that flow in the particle perpendicular to $\phi_{\mathrm{p}}$ are parallel to the coil winding and the particle surface $(A)$. The transmitter coil of the EMS is parallel to the inspection plane (Fig.3(a)). In an infinite medium the eddy currents are unbounded but their density falls with distance, as does the flux. From the Maxwell equations the solution of the density of the eddy currents $(J)$ in the $z$ direction (Fig. 3(b)), provided that they vary with the $x$ direction (depth in the inspected material), is given by [7]:

$$
J(x) / J_{0}=\exp \left(-(\omega \mu \sigma / 2)^{1 / 2} x\right) \cos \left[\omega t-(\omega \mu \sigma / 2)^{1 / 2} x\right]
$$

where $J(x) / J_{0}$ is the eddy current density ratio between depth $x$ and the surface and $\omega$ is the angular frequency $(\mathrm{rad} / \mathrm{s})$.

The standard depth of penetration $(\delta)$ of the eddy currents is defined as $1 / \mathrm{e}$ or $37 \%$ of the strength of the currents at the surface of the material [8]. For easier mathematical calculations, Hogemaier [9] defined the effective depth of penetration $\left(\delta_{\mathrm{e}}\right)$ as $3 \delta$. Inserting $\delta$ for $x$ in Eq. (3), $\delta_{\mathrm{e}}$ can be written as [9]:

$$
\delta_{\mathrm{e}}=150(1 / \sigma f \mu)^{1 / 2}[\mathrm{~cm}]
$$

Each metal has a certain $\delta_{\mathrm{e}}$ at a specific $f$ as a function of differences in $\sigma$. Using Eq. (4), $\delta_{\mathrm{e}}$ can be plotted as a function of $f$ for different non-ferrous metals. The graph in Fig. 4 shows that metals having a defined thickness $d$ can be differentiated based on differences in $\delta_{\mathrm{e}}$ at a certain frequency $f$.

$\delta_{\mathrm{e}}$ of the eddy currents depends on the electrical conductivity $\sigma$ of the inspected metal (Eq.(4)). However, $f$ has a significant influence on $\delta_{\mathrm{e}}$ into the specimen. The maximum depth of penetration is higher at lower $f$ and decreases when $f$ increases (Fig. 4).

\section{Variation of the Receiver Coil Parameters}

As mentioned before, the interaction between the metal object and the magnetic field is recorded with a receiver coil. Using the electrical circuit representation, which consists of a coil in serial with a resistor (Fig. 5(a)), the induced eddy currents into the metal 

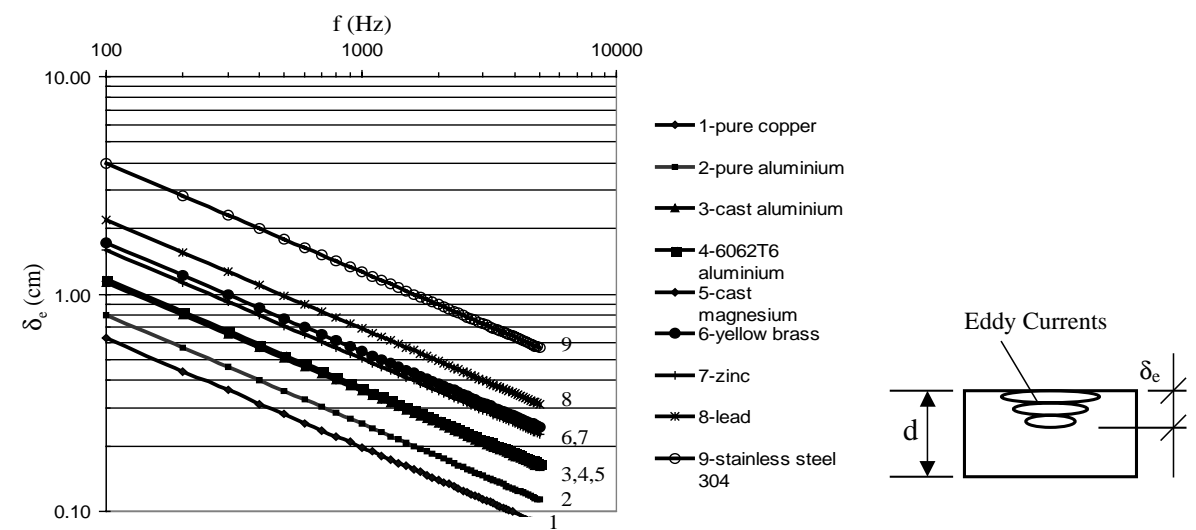

FIGURE 4 The effective depth of penetration $\delta_{\mathrm{e}}$ of the eddy currents into material as a function of frequency.



(a)

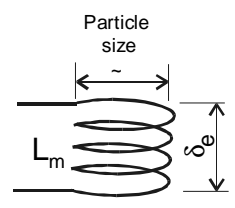

(b)

FIGURE 5 (a) A circuit representation of the eddy currents ( $M=$ mutual inductance between $L_{\mathrm{m}}$ and $L_{\mathrm{s}}$ ); (b) electrical inductance of the coil.

piece are described. The induced eddy currents can be represented as the windings of a coil having the electrical inductance $L_{\mathrm{m}}$.

$L_{\mathrm{m}}$ is modified as a result of geometric variations of the coil formed by the induced eddy currents. The coil diameter is linked to the particle size (in fact the projected particle area), while the height of the coil is correlated to $\delta_{\mathrm{e}}$ (Fig. 5(b)). Inside of an alternating magnetic field, the electrical impedance $(Z)$ defines the parameters of the coil.

The optimum frequency at which metals can be differentiated with maximum sensitivity was defined by Förster [10] as a relation between the receiver coil parameters and $\delta_{\mathrm{e}}($ Eq. (5)),

$$
f_{0}=2\left(r / \delta_{\mathrm{e}}\right)^{2}[\mathrm{~Hz}]
$$

where $r[\mathrm{~mm}]$ is the radius of the receiver coil [10].

Equation (5) helps to find the optimum frequency at which the maximum sensitivity is obtained for a specific type of the metal. The changes in $Z$ of the receiver coil are determined by the changes in the attenuation coefficient of the electromagnetic waves $(\alpha)$ in the test sample. $\alpha$ is often expressed in terms of the depth of penetration of the eddy currents. $Z_{\mathrm{s}}$ provides information about the magnitude of the eddy currents induced in the test piece, which is in this case correlated with the voltage amplitude from the receiver coil. 


\section{METHODS AND MATERIALS}

The electromagnetic sensor that was developed by Delft University of Technology in co-operation with $\mathrm{S}+\mathrm{S}$ Metallsuchgeräte und Recyclingtechnik $\mathrm{GmbH}$, is based on the theory described previously. This sensor comprises a single transmitter coil of $100 \mathrm{~mm}$ width, $680 \mathrm{~mm}$ length and has 300 turns. The receiver coils are connected into a line array. The receiver unit is linked to a personal computer via an RS 232 serial port, and the data are analysed using programs written in $\mathrm{C}++$ software.

The particles slide down a slope (Fig. 6(a)). The angle of the slope can be adjusted, which allows adjustment of the sliding velocity. The experiments were conducted with an angle of 45 degrees, which correspondes to a particle velocity between 1.3 and $1.5 \mathrm{~m} / \mathrm{s}$. Each particle provides signal amplitude $(U)$ and phase shift $(\varphi)$ to the computer (Fig. 6(b)).

The sensor uses three modules that each comprise 10 coils. The resolution of the sensor in the $x$ direction is correlated with the particle velocity. In this set-up it is about $8 \mathrm{~mm}$. A receiver coil has 2500 turns and $7 \mathrm{~mm}$ diameter, which defines the sensor resolution on the $y$ direction (Fig. 7(a)). Each receiver coil consists of two sub-coils that are connected in opposite-phase (Fig. 7(b)). The signal from these coils

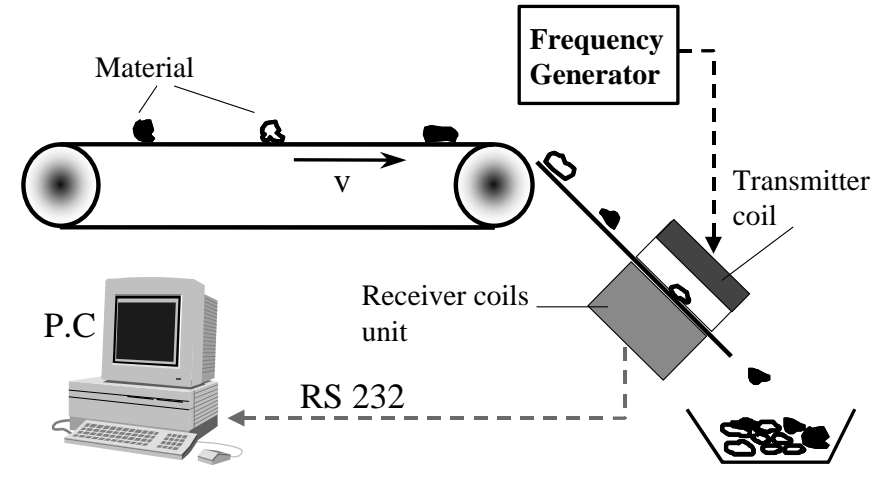

(a)

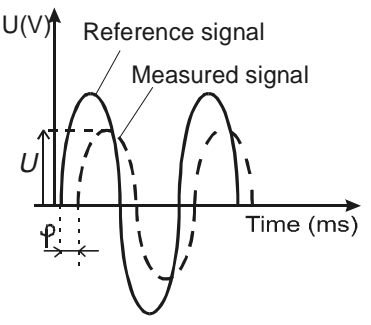

(b)

FIGURE 6 (a) The experimental set-up and (b) the measurement principle of $U$ and $\varphi$ (the reference signal is defined as the signal from the transmitter coil and the measured signal is from the receiver coils).

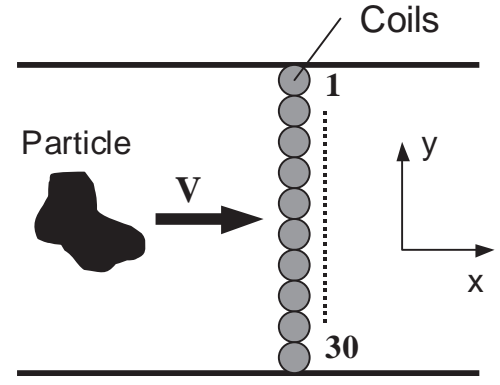

(a)

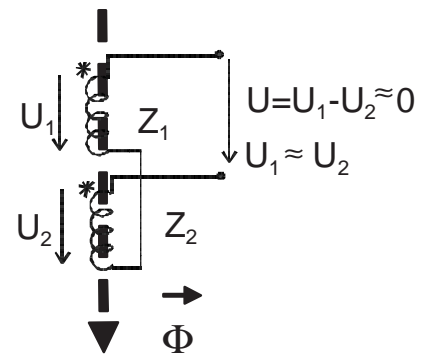

(b)

FIGURE 7 Connection of the receiver coils: (a) arrangement; (b) differential connection of the coils $\left(\mathrm{Z}_{1}\right.$ - impedance of the upper coil and $Z_{2}$ - impedance of the lower coil). 




FIGURE 8 Scrap non-ferrous metals from a shredder.

is transmitted to the computer and during its movement a particle is scanned every $5 \mathrm{~ms}$.

The experiments were carried out using particles from an industrial shredder, which cuts the particles into irregular sizes with sizes from $20 \mathrm{~mm}$ up to $150 \mathrm{~mm}$ (Fig. 8).

\section{EXPERIMENTAL RESULTS}

\section{Voltage Amplitude (U)}

For the first set of experiments, particles with low electrical conductivity such as stainless steel, are analysed and compared to particles with medium and high conductivity, such as copper, aluminium alloys, magnesium and brass. Figure 9(a) shows the distribution of $U$ for stainless steel particles at $f=700 \mathrm{~Hz}$. Each sensor provides the variation of $U$ as a function of time. The distribution of $U$ for one sensor is depicted in Fig. 9(b), where two parameters, $A_{1}$ and $A_{2}$, are defined.

Figure 10(a) illustrates the variation of $U$ for cast aluminium particles at $700 \mathrm{~Hz}$. $U$ decreases when a cast aluminium particle passes the sensor coils. Figure 10(b) presents the variation of $U$ for one receiver coil for cast aluminium.

Figure 11 shows the experimental values of $A_{2}$ obtained at three frequencies for cast aluminium, wrought aluminium, brass, magnesium and stainless steel. Stainless steel can be more easily distinguished from other metals at $700 \mathrm{~Hz}$. At the same frequency a slight differentiation is visible between cast aluminium, wrought aluminium on one side and brass and magnesium on the other. A stronger attenuation of $U$ for stainless steel is obtained at higher frequencies.

For metals such as cast and wrought aluminium at $700 \mathrm{~Hz}$, no significant differences in $U$ can be recorded. Improvements in detection are possible by applying a multifrequency operation. For example, three frequencies of the transmitter coil signal could be implemented at $700 \mathrm{~Hz}, 1.5 \mathrm{kHz}$ and $5 \mathrm{kHz}$.

Additional experiments were carried out for brass with nickel coating and stainless steel. Coated brass particles occur in metal streams from several typical home appliances, for example taps or pipes. Cameras are unable to detect differences between these metals and stainless steel, because they have approximately the same colour. 


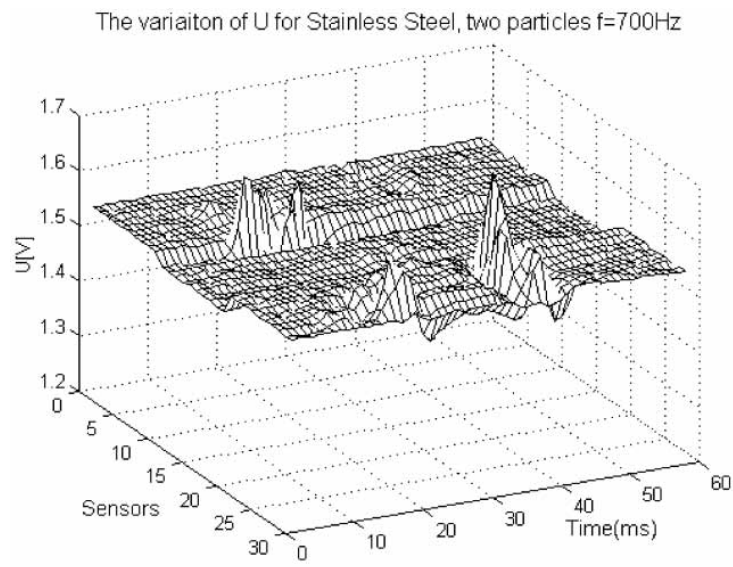

(a)



(b)

FIGURE 9 (a) $U$ for stainless steel at $700 \mathrm{~Hz}$; (b) the variation of the voltage $U$ for stainless steel - one receiver coil.

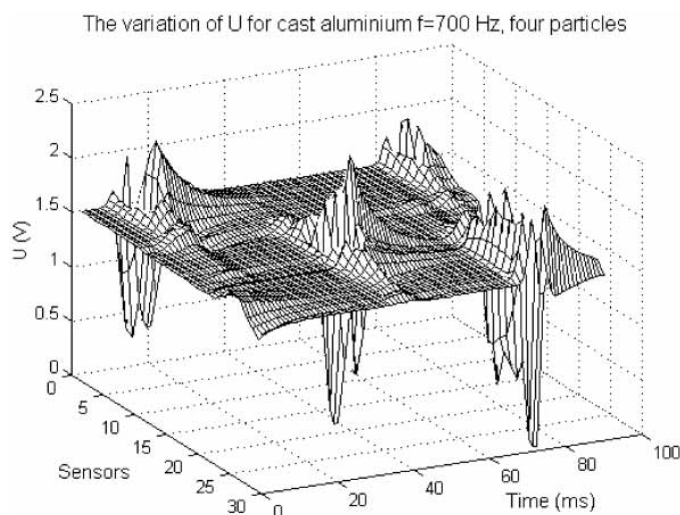

(a)

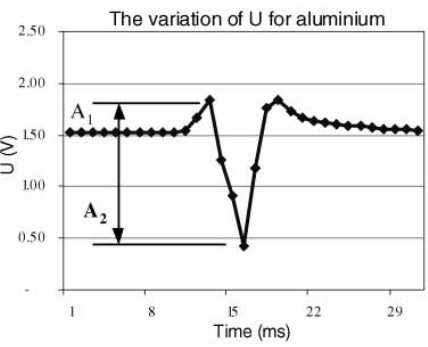

(b)

FIGURE 10 (a) The voltage amplitude for cast aluminium at $f=700 \mathrm{~Hz}$; (b) the variation of the voltage for a single receiver coil.

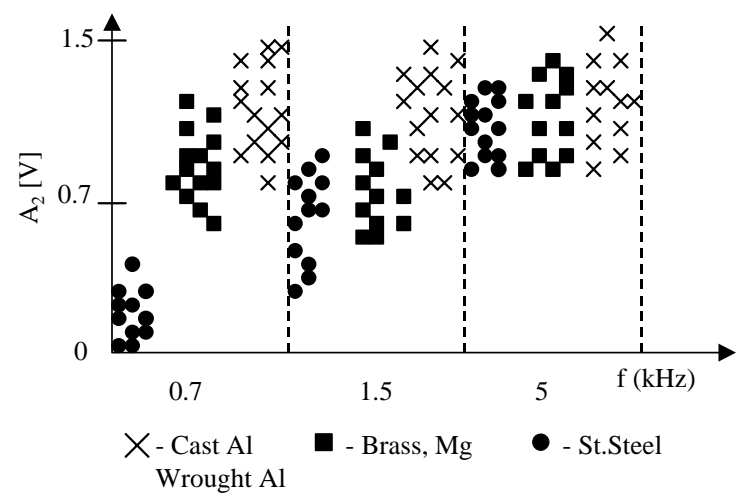

FIGURE 11 Variation of $A_{2}$ at different frequencies. 


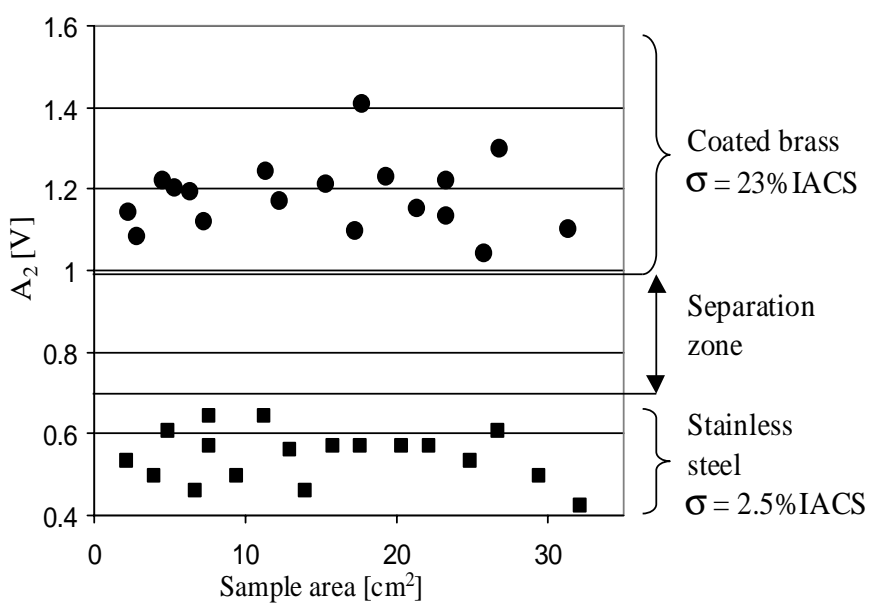

FIGURE 12 Differentiation between stainless steel and coated brass particles.

In Fig. 12 the results for $A_{2}$ obtained for stainless steel and coated brass particles are shown.

Copper is highly conductive compared to brass, which is a medium conductive metal (Fig. 2). Because of higher conductivity the experiments carried out with copper particles showed that voltage of copper was more affected in amplitude than that of brass. Additionally, $A_{1}$ is much higher in amplitude for copper compared to brass. The thickness of the copper used for these experiments was more than $3 \mathrm{~mm}$. In this case the eddy currents induced in the copper are more uniform, due to the fact that $\delta_{\mathrm{e}}$ is less than the minimum particle thickness [7].

\section{Phase Shift $(\varphi)$}

The second parameter that has been recorded from the receiver coils is $\varphi$. This represents the phase difference between the transmitter and the receiver coil signals. If there is no metal present above the receiver coils, the sensor provides approximately zero degree phase shift (Fig. 7(b)). When a metal is close to the receiver unit, $\varphi$ is modified as a function of $\sigma$ and the particle geometry parameters, e.g. $d$ and $A$. In earlier work it was found that $\varphi$ has a maximum at a specific frequency [1]. This maximum also depends on the particle geometry, especially on particle thickness.

Shredded particles have many shapes, so supplementary information about the particle geometry improves the identification. In addition, the localisation of particles on a conveyer belt is easier. The localisation of a particle is necessary for the ejection system [11].

Figure 13 shows the variation of $\varphi$ for a brass particle. Each square of the vertical axis represents a receiver coil. For these experiments 30 receiver coils are connected to a data processing unit.

The variation of the phase shift $\varphi$ depends not only on $A$ but also on $d$ and $f$. Figure 14 represents the variation of $\varphi$ for aluminium particles, which have the same $A$ but different $d$ at $f=700 \mathrm{~Hz}$. 


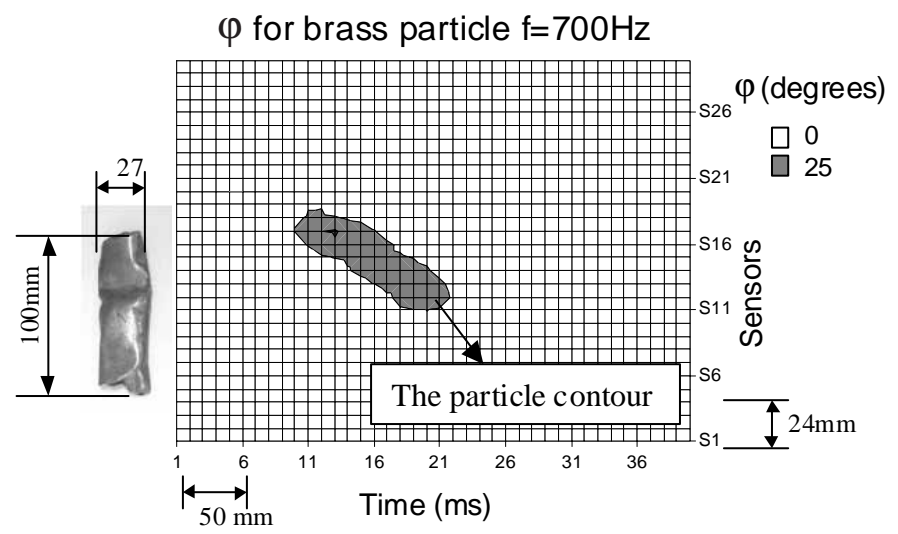

FIGURE 13 The phase shift $\varphi$ variation for a brass particle at $700 \mathrm{~Hz}$. The particle velocity is about $1 \mathrm{~m} / \mathrm{s}$.



FIGURE 14 The phase shift $\varphi$ variation for aluminium particles for different thickness $d$.

\section{DISCUSSION}

\section{Voltage Amplitude ( $U$ )}

When there is no material present, the sensor provides the voltage amplitude $U$ of approximately $1.5 \mathrm{~V}$. The voltage amplitude increases when a stainless steel particle crosses the line of the receiver coils, because the eddy currents induced into the material generate a magnetic field in the same direction as the normal field created by the transmitter coil. For stainless steel, $U$ is less affected in amplitude than for other non-ferrous metals.

The distribution of the voltage amplitude for each receiver coil is dependent on $\sigma$, but also depends on the particle geometry. As can be seen in Fig. 10(a), the distribution of $U$ comprises different peaks for each sensor element. $A_{2}$ represents the lower peak for the stainless steel particles, which is the most important part for analysing variations in $U$. The minimum of these peaks for aluminium are in the centre of the particle due to the fact that the induced eddy currents are more uniformly distributed than at the extremities (the edge effect) [10]. The edge effect produces lower peaks for stainless 
steel, which are defined by $A_{2}$ (Fig. 9(b)). The variation of the voltage amplitude for stainless steel differes from that of aluminium.

The experiments showed that the variation of $U$ presented in Fig. 10(b) is the same for other non-ferrous metals such as copper, wrought aluminium, magnesium and brass. Figures 9(b) and 10(b) show the principle of differentiation between stainless steel and other non-ferrous metals, where $A_{2}$ is the main parameter of the differentiation.

Experiments showed that at frequencies less than $1 \mathrm{kHz}$ good results are obtained for stainless steel differentiation. However, $700 \mathrm{~Hz}$ is the frequency where also high conductive metals like copper and aluminium alloys could be distinguished from medium conductive metals such as brass and bronze.

Using Eq. (5) and the coil parameters of the experimental set-up presented in Section 3, it was found that the maximum sensitivity for the separation between different metals is obtained at $f=700 \mathrm{~Hz}$. The same frequency was used in the experiments, which demonstrates that at low frequency metals are better distinguished from each other.

Due to the fact that stainless steel has an electrical conductivity much lower than brass, a thin nickel layer on brass particles does not affect the response of the sensor. This layer is mostly between $10-20 \mu \mathrm{m}$ thick and most particles have a thickness of more than $7 \mathrm{~mm}$. However, the weak signal from the stainless steel particles provides a clear differentiation between the two types of metals based on variations in $A_{2}$ (Fig. 12).

Concluding the analysis of the voltage amplitude, highly conductive particles show a stronger attenuation of $U$ compared to medium and low conductive particles. The greater $\sigma$, the more is $U$ attenuated at the output of the receiver coils. Stainless steel has a different distribution of $U$, with good results for the separation of this metal from other non-ferrous particles. At $700 \mathrm{~Hz}$, however, the sensor works at maximum sensitivity and experiments show that metals can be better distinguished than at other frequencies.

The geometry of the particle also affects the response of the sensor, particularly when particles have close electrical conductivities. Large particles, with $A$ more than $25 \mathrm{~cm}^{2}$, have more influence on the greater sensor output $(U$ and $\varphi)$ than small particles with $A$ less than $10 \mathrm{~cm}^{2}$. In comparison, for those particles which have high and low electrical conductivities the particle geometry has not much influence on $U$ and $\varphi$, because electrical conductivity provides a stronger signal to the sensor.

\section{Phase Shift $(\varphi)$}

After scanning, the contour of a particle is clearly seen by the variation of the phase shift, and therefore the area $A$ can be computed. The accuracy of the computed area is approximately $\pm 5 \%$ compared to the real area, which has been determined by manual measurement.

By combining $A$ and $d$ from the phase shift, a good approximation of the particle geometry can be obtained. This information combined with the amplitude variation of the voltage improves the differentiation between particles, especially for those having close electrical conductivities. Beside that, the experiments show that small particles having high conductivity give approximately the same voltage as large particles with medium conductivity. Additional information about the particle geometry leads to a better differentiation and particle localisation. 


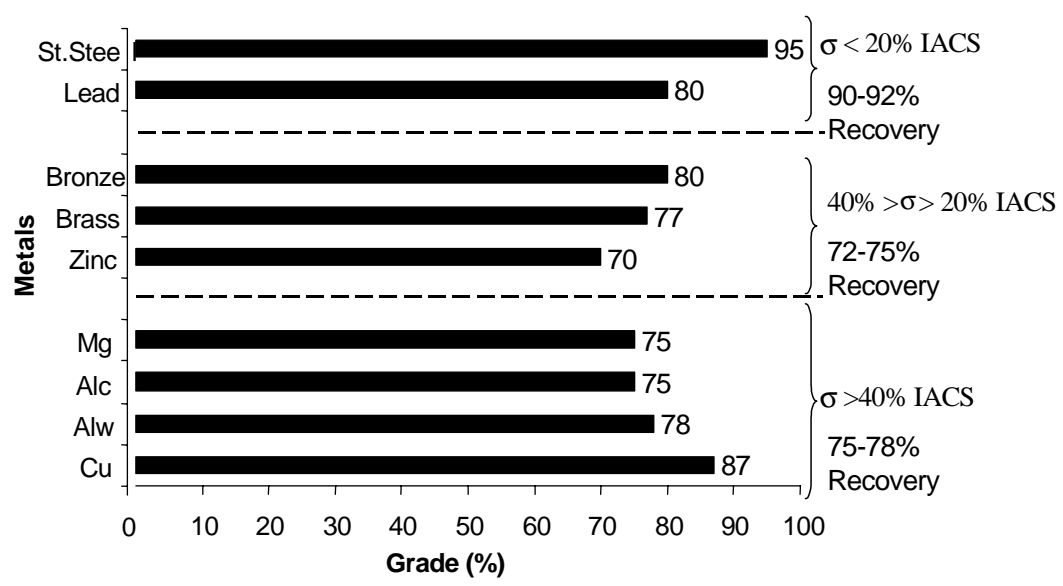

FIGURE 15 Differentiation of non-ferrous metals using EMS at $700 \mathrm{~Hz}$.

\section{Separation Between Various Non-Ferrous Metals}

Based on the variation of the voltage, different non-ferrous metals can be classified into three main groups: with high, medium and low conductivity. For those particles with the same voltage, $\varphi$ was analysed regarding the inspected particle area. The frequency at which the EMS works plays a crucial role in the differentiation between non-ferrous metals. The experiments showed that at $700 \mathrm{~Hz}$ better results of the distinction between non-ferrous metals are obtained than at other frequencies (Fig. 11). In Fig. 15 the results obtained for the separation of several non-ferrous metals at $700 \mathrm{~Hz}$ are presented. Very good results are recorded for the grade and the recovery of weakly conducting metals. However, metals of the medium and high conductivity show good results for the grade but a lower recovery compared to metals with low conductivity.

\section{CONCLUSIONS}

A method for metal characterisation and separation using an EMS based on the eddy current principle is presented. The results demonstrate that the method can be used in applications for scrap non-ferrous metals identification, separation and quality control.

An advantage is the contact-free and rapid inspection of the material. Good results are obtained for the differentiation of weakly conducting metals, e.g. stainless steel, lead, from highly conductive, e.g. copper, aluminium alloys, magnesium. Using low and high frequencies simultaneously, metals are better distinguished, especially those that have close conductivity. The EMS can be integrated into an industrial system, comprising data processing units and ejection systems.

The electromagnetic sensors are not sensitive to dust, coatings or other impurities covering the metal particles. Pre-processing steps such as particle cleaning are not necessary. The sensors can operate post shredding and the output of the sensor is estimated between 6 to 8 tonne/h for a sensor width of $1 \mathrm{~m}$ at a particle velocity around $1 \mathrm{~m} / \mathrm{s}$. 


\section{NOMENCLATURE}

$A\left[\mathrm{~m}^{2}\right]=$ particle area

$A_{1}[\mathrm{~V}]=$ voltage variation $($ above $1.5 \mathrm{~V})$

$A_{2}[\mathrm{~V}]=$ voltage variation (below $1.5 \mathrm{~V}$ )

$a\left[\mathrm{~m}^{2}\right]=$ area of integration

$B[\mathrm{~T}]=$ magnetic induction

$d[\mathrm{~m}]=$ particle thickness

$D[\mathrm{~m}]=$ receiver coil diameter

ECS $=$ eddy current separator

EMS $=$ electromagnetic sensor

$f[\mathrm{~Hz}]=$ frequency

$H[\mathrm{~A} / \mathrm{m}]=$ magnetic field intensity

$J[\mathrm{~A} / \mathrm{m}]=$ eddy current density

$J_{0}[\mathrm{~A} / \mathrm{m}]=$ surface eddy current density

$L_{\mathrm{m}}[\mathrm{H}]=$ coil inductance (metal piece)

$L_{\mathrm{S}}[\mathrm{H}]=$ receiver coil inductance

$M[\mathrm{H}]=$ mutual inductance

$N_{\mathrm{s}}=$ number of turns in the receiver coil

$r[\mathrm{~mm}]=$ radius of the receiver coil

$R[\Omega]=$ receiver coil resistance

$U[\mathrm{~V}]=$ receiver coil voltage amplitude

$v[\mathrm{~m} / \mathrm{s}]=$ particle velocity

$V_{\mathrm{p}}[\mathrm{V}]=$ voltage excitation of the primary coil

$x[\mathrm{~m}]=$ depth of eddy currents below the metal surface

$Z[\Omega]=$ electrical impedance

$X_{\mathrm{L}}[\Omega]=$ receiver coil reactance

$\delta[\mathrm{m}]=$ standard depth of penetration of eddy currents

$\delta_{\mathrm{e}}[\mathrm{m}]=$ effective depth of penetration of eddy currents

$\phi[\mathrm{Wb}]=$ flux intensity

$\phi_{\mathrm{E}}[\mathrm{Wb}]=$ equilibrium flux intensity

$\phi_{\mathrm{p}}[\mathrm{Wb}]=$ primary flux intensity

$\phi_{\mathrm{s}}[\mathrm{Wb}]=$ secondary flux intensity

$\varphi[$ degrees $]=$ phase shift

$\mu[\mathrm{H} / \mathrm{m}]=$ magnetic permeability

$\mu_{0}[\mathrm{H} / \mathrm{m}]=$ vacuum magnetic permeability $\left(4 \pi \times 10^{-7}\right)$

$\mu_{\mathrm{r}}[\mathrm{H} / \mathrm{m}]=$ relative magnetic permeability

$\sigma\left[\Omega \mathrm{m}^{-1}\right]=$ electrical conductivity

$\omega[\mathrm{rad}]=$ angular frequency

\section{References}

[1] A. Nijkerk, W.L. Dalmijn, Handbook of Recycling Techniques, 5th Edn., The Hague, 2001.

[2] A. Rosenfeld, A. Gesing and B. Farachbakhsh, Sorting of Aluminium Alloy Scrap by Laser Induced Optical Emission Spectroscopy, Third International Symposium on Recycling of Metals and Engineering Materials, Las Vegas, Nevada, USA, TMS 1995. 
[3] US Patent 1997, no.736275, System for Sorting Scrap Particles Based upon their Color.

[4] T.P.R de Jong, W.L. Dalmijn, X-ray Transmission Imaging for Process Optimisation of Solid Resources, Proceedings R'02 Congress, Recovery Recycling Re-integration, Geneva, Switzerland, February 12-15, 2002.

[5] M.B. Mesina, T.P.R. de Jong, H.U.R. Kattendidt, W.L. Dalmijn, Non-Ferrous Metals Characterisation and Identification using an Electromagnetic Sensor, Proceedings R'02 Congress, Recovery Recycling Re-integration, 2002, Geneva, Switzerland, February 12-15.

[6] D.E. Bray, Non-Destructive Evaluation - A Tool in Design, Manufacturing and Service, Revised Edn., CRC Press, 1997.

[7] N. Ida, Numerical Modelling for Electromagnetic Non-Destructive Evaluation, Charman and Hall, USA, 1995.

[8] J. Cox, Non-Destructive Testing -Eddy Current, PHDiversified, INC Publisher, UK, 1997.

[9] D. Hogemaier, Handbook of Nondestructive Testing, 2nd Edn., Vol.4, American Society for Nodestructive Testing, Columbus, USA, 1987, Section 14.

[10] F. Förster, Handbook of Non-destructive Testing, Vol. 4, 2nd Edn., Colombus OH, 1986, Section 6.

[11] H.U.R. Kattendidt, T.P.R. de Jong, W.L. Dalmijn, (2003). Multi-Sensor Identification and Sorting of Bulk Solids, Control Engineering Practice, 44, 41-47.
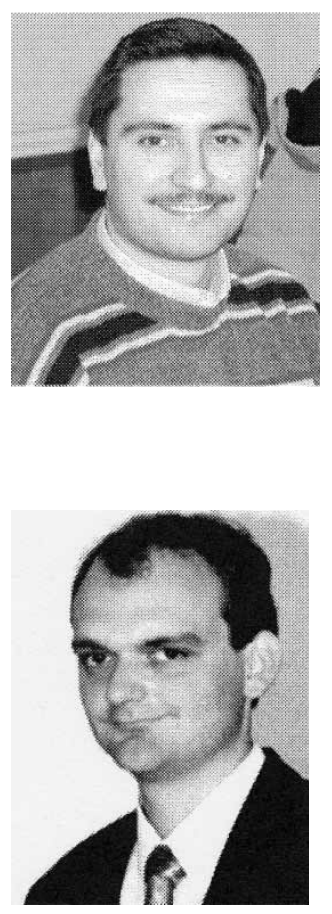

Marian B. Mesina was born in 1972. He graduated from "Politehnica" University of Bucharest in 1996. In 2000 he joined Delft University of Technology as a Ph.D. student. His main area of interest is the development of new techniques based on sensor technology for separation and sorting of secondary raw materials.

Tako Pieter Rinze de Jong obtained in 1993 his M.Sc. (Ir.) in applied earth sciences from the Delft University of Technology. Until 1998 he worked as a researcher at the Faculty of Applied Earth Sciences. In 1998 and 1999 he was associated with the Institute of Environmental Energy and Process Innovation in Apeldoorn working on design of the recycling and waste processing systems. In 1999 he obtained his Ph.D. degree from the Delft University of Technology and joined the Faculty of Applied Earth Sciences as a project manager. His main area of interest is sensor sorting and automatic quality control. 


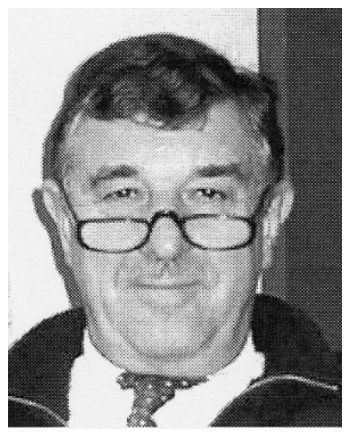

Wijnand L. Dalmijn graduated from the Delft University of Technology on the subject of the recovery of metals from incinerator residues. During the period of 1974 to 1980 he worked on numerous projects concerning the recovery of materials from waste streams. As a technical manager of Maltha Int. B.V. he was involved in the design of automated sorting plants for glass recycling. During his association with Alcan Int. Ltd., MG Frankfurt and other companies research into metal sorting was conducted. In 1991 he joined the Delft University of Technology as a professor and in 1999 he was pointed the Dean of the Faculty of Design, Engineering and Production. 